\title{
Análisis de indicadores ambientales espacio-temporales de agua, suelo y vegetación
}

\author{
María Josefa Jiménez-Moreno ${ }^{1 \S}$ \\ Rodrigo Rodríguez-Laguna ${ }^{2}$ \\ Miguel Jorge Escalona-Maurice ${ }^{3}$ \\ Ramón Razo-Zarate ${ }^{2}$ \\ Otilio Arturo Acevedo-Sandoval ${ }^{2}$
}

${ }^{1}$ Instituto de Ciencias Básicas e Ingeniería-Universidad Autónoma del Estado de Hidalgo-Ciudad del Conocimiento. Carretera Pachuca-Tulancingo km 4.5, Mineral de la Reforma, Hidalgo. CP. 42184. Tel. 55 58045900, ext. 1843. ${ }^{2}$ Instituto de Ciencias Agropecuarias-Área Académica de Ciencias Agrícolas y Forestales-Universidad Autónoma del Estado de Hidalgo. Avenida Universidad km 1, Rancho Universitario Exhacienda de Aquetzalpa, Hidalgo. AP. 32. CP. 43600. Tel. 771 7172000, ext. 2431 y 2901. (rlaguna@uaeh.edu.mx; rrazo29@yahoo.com.mx; acevedo@uaeh.edu.mx). ${ }^{3}$ Desarrollo Rural-Campus Montecillo-Colegio de Postgraduados. Carretera México-Texcoco km 36.5, Montecillo, Texcoco, Estado de México. CP. 56230. Tel. 595 9520200, ext. 1843. (mescalona@colpos.mx).

${ }^{\S}$ Autora para correspondencia: jimory6@gmail.com.

\section{Resumen}

Los indicadores ambientales son de suma importancia porque proveen información cuantitativa y cualitativa para evaluar el uso, disponibilidad y alteración de los recursos naturales. El presente ensayo tuvo como objetivo revisar del año 2006 a 2018 indicadores ambientales con un enfoque espacial y temporal, utilizados para evaluar la condición del ambiente. Posteriormente, se clasificó la información consultada en tres grupos principales de indicadores de agua, suelo y vegetación. Después se determinó las potencialidades de los diferentes métodos de indicadores ambientales, dentro de estos hallazgos relevantes se observó que cada metodología depende de la calidad de la información y de los objetivos de cada estudio. Dentro de las metodologías analizadas las de mayor relevancia por la precisión de sus resultados obtenidos, fueron los índices de vegetación y análisis multicriterio, ya que permite percibir explícitamente los parámetros de la zona que han sido modificados por actividades humanas. Se concluye que las metodologías de indicadores ambientales dependen del aspecto espacio-temporales y la calidad de los datos, así como del recurso natural a evaluar.

Palabras clave: análisis multicriterio, escala de análisis, índices de vegetación, metodologías, recursos naturales.

Recibido: septiembre de 2019

Aceptado: octubre de 2019 
Los problemas ambientales afectan seriamente los recursos naturales debido las actividades de los seres humanos (Walz, 2015). En la literatura revisada, se ha demostrado que los indicadores ambientales sirven para evaluar la 'salud' de los ecosistemas (Luo, Huai y Gao, 2017) debido a que son instrumentos fundamentales para generar, almacenar y analizar información (Rodríguez et al., 2013; Schindler et al., 2015; Gao et al., 2017).

Un indicador describe situaciones de un espacio en un momento dado, es decir son indicadores espacio-temporales (Nowak y Schneider, 2017; Pratama et al., 2017; Bao y Zou, 2018) que proveen, condesan, sistematiza y ordenan información cuantitativa o cualitativa (Schindler et al., 2015; Li et al., 2016; Bao y Zou, 2018). Los indicadores ambientales presentan directrices, estadísticas, categorías clasificatorias y situaciones predictivas, que brindan señales de posibles escenarios futuros (Rodríguez et al., 2013; Wen et al., 2017) los cuales deben ser prácticos y realistas (Walz, 2015; Turner et al., 2016).

La presente revisión aporta una clasificación de tres grupos principales de indicadores ambientales de agua, suelo y vegetación, así como una base conceptual espacio-temporal aplicados para la visualización, evaluación y modelación de datos multipropósito hacia la toma de decisiones (Xu y Zhang, 2013; Klug y Kmoch, 2015; Echavarren, 2017).

\section{Indicadores ambientales espacio temporales}

La intención de este documento no es abrir una discusión profunda y detallada acerca de los planteamientos teóricos y prácticos referentes a los indicadores ambientales, más bien es mostrar diferentes trabajos en donde se ha utilizados indicadores ambientales espacio-temporales para conocer la situación del ambiente en el que se aplicó las diferentes metodologías para evaluar la situación de los ecosistemas (Bredemeier et al., 2015; Turner et al., 2016; Dyer et al., 2017; Zhang y Xu, 2017).

Indiscutiblemente los indicadores ambientales espacio-temporales es un tema amplio y diverso, que los autores los han usado para evaluar los ecosistemas, su dinámica, así como las aplicaciones de gestión ambiental (Luo et al., 2017).

Investigaciones recientes muestran que los indicadores ambientales presentan datos y comparaciones útiles para contribuir en la sostenibilidad de la naturaleza, en la toma de decisiones y formulación de políticas ambientales (Alphan, 2017; Neher et al., 2017; Nowak y Schneider, 2017; Pratama et al., 2017) y constituyen una herramienta básica de información y seguimiento de los procesos que se originan en el medio natural, social y económico (Xu y Zhang, 2013; AsumaduSarkodie y Owusu, 2017; Sinha et al., 2017).

Los indicadores ambientales dan seguimiento al impacto ambiental biofísico y socioeconómico, reconocidos como una necesidad fundamental a nivel local, regional, nacional e incluso internacional (Neher et al., 2017; Sinha et al., 2017). Evalúa el impacto ambiental mediante herramientas innovadoras y precisas para predecir las consecuencias del desarrollo (Klug y Kmoch, 2015; Arnaiz-Schmitz et al., 2017; Han et al., 2017) proporcionando información útil para la planificación al presentar datos y comparaciones actualizados (Alphan, 2017; Gao et al., 2017; Luo et al., 2017; Bao y Zou, 2018). 
Con base a lo anterior, se determinó que los indicadores ambientales son herramientas básicas de información y seguimiento de procesos que se dan en el medio natural, social y económico, para conocer el estado y la tendencia de los recursos naturales (Xu y Zhang, 2013; Asumadu-Sarkodie y Owusu, 2017; Sinha et al., 2017). Además, proporcionan información de forma sencilla y fácil de entender estadísticamente; a través, de un modelo o conjunto de supuestos que relacionan el indicador con fenómenos más complejos (Walz, 2015; Neher et al., 2017) comunicando información científica y técnica sustentada en la problemática ambiental (Schindler et al., 2015; Turner et al., 2016).

Por lo tanto, los indicadores ambientales enfocados a los recursos naturales miden y evalúan la calidad del ambiente (Walz, 2015; Shrestha et al., 2017) definen el uso y aprovechamiento de estos, con la finalidad de disminuir la contaminación o degradación de la naturaleza (Park et al., 2015; Neher et al., 2017; Nowak y Schneider, 2017).

\section{Aplicaciones de indicadores ambientales}

Debido a que la información utilizada para construir indicadores ambientales espacio-temporales es amplia y diversa, se requiere de un marco conceptual que permita estructurar la información y facilidad de acceso e interpretación (Azadeh et al., 2017; Bao y Zou, 2018), siendo indispensable revisar y evaluar diferentes trabajos sobre indicadores ambientales para conocer la importancia de los recursos naturales en un análisis espacio-temporal de cada estudio y definir las metodologías de mayor eficacia (Luo et al., 2017; Nowak y Schneider, 2017). Con base a las características de cada método, se clasificó la información consultada en tres grupos principales: indicadores ambientales de agua, suelo y vegetación.

\section{Indicadores de agua}

El agua es esencial para garantizar la integridad y sostenibilidad de los ecosistemas terrestres (WWAP, 2017), siendo uno de los recursos más importantes para la vida en el planeta ya que todos los seres vivos, incluyendo el ser humano, depende de su disponibilidad para su funcionamiento. A pesar de su importancia, es un recurso escaso, amenazado y en peligro se ha incrementado la extracción, contaminación y acceso al agua (Vollmer et al., 2016; WWAP, 2017).

De los diferentes trabajos de indicadores ambientales enfocados al estudio del agua Klug y Kmoch (2015) generaron, evaluaron y modelaron datos multipropósitos de indicadores con la finalidad de realizar acciones inmediatas en tiempos de crisis para monitorear los flujos y extracciones de agua con información espacial en una escala de análisis global Describieron nuevas formas de procesamiento de indicadores utilizando redes de sensores inalámbricos y servicios web estandarizados.

La información ambiental estandarizada se incorporó automáticamente a los servicios de procesamiento web para la entrega oportuna de información, descubrimiento y acceso a las condiciones ambientales espaciales. Dicho monitoreo de flujos y extracciones de agua se realizó con la finalidad de asegurar el suministro oportuno de información espacial para reducir los tiempos de demora en el abastecimiento de agua. 
En tanto, Vollmer et al. (2016) desarrollaron y evaluaron índices que miden la salud ecológica del agua, observando que a medida que los factores de estrés en los sistemas de agua aumentan en magnitud y abundancia, las necesidades informativas y la capacidad de administrar los sistemas de agua cambian según la escala espacial de análisis (local a una global). Por lo que determinaron que los indicadores cuantitativos son un medio común para evaluar las dimensiones de un sistema de agua y aporta conocimientos científicos para los responsables de la toma de decisiones y políticas.

Por su parte Gao et al. (2017) analizaron indicadores ambientales en una escala espacial regional de la precipitación, evapotranspiración y observaron que la precipitación se incrementa significativamente. Sin embargo, la evapotranspiración demostró una tendencia decreciente no significativa. Mientras que, la precipitación mostró una evidente evolución, crecimiento y tendencia en el cambio del clima según el modelo.

Con base a lo anterior, los indicadores permitieron determinar que los recursos hídricos aumentarán con el calentamiento global. Desde la perspectiva temporal, presentó una tendencia ligeramente decreciente en la precipitación y un aumento significativo desde la perspectiva espacial.

\section{Escala regional}

En tanto Shrestha et al. (2017) Utilizaron indicadores en una escala de análisis espacial regional con un análisis de varianza para reducir los impactos ambientales y mejorar la calidad del agua relacionada con los nutrientes en los humedales por actividades agrícolas, detectando muchas interacciones significativas de tiempo y por tipo de flujo en las áreas de humedales, así como varios parámetros y puntajes de hábitat totales que mostraron una mejora en general de los humedales a diferencia de las áreas agrícolas. Los parámetros del hábitat evaluados mejoraron significativamente los humedales, aunque no siempre de manera constante durante todo el año.

Bao y Zou (2018) en una escala de análisis espacial regional evaluaron la dinámica integral de la variabilidad temporal y espacial del agua. Aplicaron un sistema de evaluación integral y dinámico determinado por umbrales y normas a través de una estructura jerárquica para calcular ponderaciones y establecer un enfoque de objetivos múltiples; a través, de valores normalizados de las características espacio-temporales entre la escasez de agua y las actividades humanas.

Los cambios espaciales y temporales de los indicadores del agua analizados presentan una gran disparidad en las relaciones entre el hombre y el agua, la cual está determinado por la escasez de agua y las actividades humanas a través del tiempo (Cuadro 1).

Cuadro 1. Indicadores ambientales del agua.

\begin{tabular}{clll}
\hline $\begin{array}{c}\text { Escala de } \\
\text { análisis }\end{array}$ & \multicolumn{1}{c}{ Datos espaciales } & \multicolumn{1}{c}{ Método empleado } & Autor \\
\hline Regional & $\begin{array}{l}\text { Región natural y grado de } \\
\text { aprovechamiento eficiente de los } \\
\text { recursos hídricos }\end{array}$ & $\begin{array}{l}\text { Modelo analítico de } \\
\text { proceso de jerarquía }\end{array}$ & $\begin{array}{l}\text { Bao y Zou } \\
(2018)\end{array}$ \\
Global & $\begin{array}{l}\text { Redes inalámbricas de sensores y } \\
\text { servicios web estandarizados }\end{array}$ & $\begin{array}{l}\text { Modelado multipropósito } \\
\text { en tiempo real }\end{array}$ & $\begin{array}{l}\text { Klug y } \\
\text { Kmoch } \\
(2015)\end{array}$ \\
\hline
\end{tabular}




\begin{tabular}{llll}
\hline $\begin{array}{c}\text { Escala de } \\
\text { análisis }\end{array}$ & \multicolumn{1}{c}{ Datos espaciales } & \multicolumn{1}{c}{ Método empleado } & \multicolumn{1}{c}{ Autor } \\
\hline Regional & $\begin{array}{l}\text { Monitoreo mensual de las corrientes de } \\
\text { agua de la cuenca } \\
\text { L5 índices de agua }\end{array}$ & Análisis de varianza & $\begin{array}{l}\text { Shrestha } \text { et } \\
\text { al. } \text { (2017) }\end{array}$ \\
& & $\begin{array}{l}\text { Evaluación de riesgos, } \\
\text { fuerzas motrices- } \\
\text { presiones-estado- } \\
\text { impacto-respuesta } \\
\text { (DPSIR) }\end{array}$ & $\begin{array}{l}\text { Vollmer } \text { et } \\
\text { al. }(2016)\end{array}$ \\
Regional & $\begin{array}{l}\text { Precipitación, evapotranspiración } \\
\text { potencia, índice de evapotranspiración } \\
\text { de precipitación estandarizada }\end{array}$ & Modelo de comparación & $\begin{array}{l}\text { Gao } \text { et al. } \\
(2017)\end{array}$ \\
\hline
\end{tabular}

*= Global, regional, local y paisaje.

\section{Indicadores del suelo}

El suelo es un recurso natural y vital para el ser humano pero debido a las actividades humanas se ha alterado la autorregulación y equilibrio, ocasionando serios problemas ambientales (Yu y Xudong, 2016; Azadeh et al., 2017) como la cantidad, calidad, bienes y servicios (Xu y Zhang, 2013). Los indicadores ambientales del suelo determinan los cambios espacio-temporales (Xu y Zhang, 2013; Zolekar y Bhagat, 2015) con la finalidad de orientar las actividades del ser humano para un equilibrio con la naturaleza.

Respecto a los indicadores ambientales del suelo un trabajo relevante es de Ligmann-Zielinska y Jankowski (2014) en el que describen una escala de análisis espacial local y multicriterio la sensibilidad espacio-temporal para evaluar la aptitud e idoneidad del suelo mediante ponderaciones que expresan distribuciones de probabilidad del hábitat caracterizando para definir la inclusión y exclusión de áreas con aptitud en el uso de la tierra.

Alphan (2017) por su parte, en una escala espacial de análisis del paisaje y con la metodología de índice de parche, definió directrices para conocer los cambios enfocados a las áreas agrícolas y zonas urbanas, destacando una gran diversidad espacial, estacional y temporal. Determinando que las formas y tamaños de cada variable de las diferentes categorías de cobertura del suelo aumentan la diversidad espacial, así como la rotación de los cultivos y el cambio en la fenología de la vegetación tienen impacto en la diversidad estacional.

Por lo que, la comprensión adecuada de las características espaciales y temporales de este atributo ambiental es de vital importancia ya que, revela las causas de los problemas ambientales y hace frente a las consecuencias de la mala gestión del suelo.

En tanto, Asumadu-Sarkodie y Owusu (2017) mediante un modelo de regresión lineal, definieron el impacto del cambio de uso del suelo a través de los indicadores ambientales en una escala de análisis local, los cuales juegan un papel importante en la sustentabilidad del suelo. Sin embargo, los métodos y prácticas agrícolas que parecen insostenibles aún desempeñan un papel fundamental en las comunidades agrícolas. Por lo tanto, la introducción de prácticas agrícolas modernas en las comunidades locales y regionales a través de la creación de conciencia contribuiría en la lucha contra el cambio climático. 
Nowak y Schneider (2017) por su parte, identificaron áreas amenazadas y degradadas por la actividad agrícola a través de indicadores ambientales en una escala de análisis regional, definida por la erosión del suelo, pérdida de nutrientes y contaminación del agua subterránea. Los resultados muestran que las áreas más amenazadas por la actividad agrícola están ubicadas en la región montañosa, mientras que la mayoría de las zonas degradadas se ubicaron en los fondos de los valles y en las áreas con agricultura intensiva.

La pendiente y el uso de la tierra también jugó un papel importante en el caso de pérdida de suelo y nutrientes. Este método proporcionó datos espacialmente específicos sobre las áreas afectadas por los procesos de degradación y determina que le suelo es un buen indicador para conocer los problemas ambientales.

En tanto que Yu y Xudong (2016); a través, de indicadores ecológicos en una escala de análisis regional, establecieron la idoneidad del suelo mediante la sensibilidad ecológica, cobertura de la vegetación, calidad del suelo y el ambiente atmosférico, entre otros factores. De acuerdo con la situación del suelo, encontraron que la elección de dichos indicadores es el factor más común e integral para la protección de áreas ecológicas, de la vegetación, el suelo, aire, agua, etc., por su frágil situación ambiental. Por lo tanto, los indicadores ambientales desempeñan un papel importante en el establecimiento del sistema de índice de evaluación de la aptitud del suelo.

De los diferentes indicadores ambientales revisados se observó la importancia de contar con indicadores del suelo espacio-temporales para evaluar los problemas ambientales y apoyar en la toma de decisiones (Cuadro 2).

Cuadro 2. Indicadores ambientales del suelo.

\begin{tabular}{|c|c|c|c|}
\hline $\begin{array}{l}\text { Escala de } \\
\text { análisis }\end{array}$ & Datos espaciales & Método empleado & Autor \\
\hline Paisaje & $\begin{array}{l}\text { Cambios espacio-temporales de la } \\
\text { cobertura del suelo }\end{array}$ & Índice de parche (LPI) & Alphan (2017) \\
\hline Local & Cambio de uso de suelo & $\begin{array}{l}\text { Modelo de regresión } \\
\text { de mínimos cuadrados }\end{array}$ & $\begin{array}{l}\text { Asumadu- } \\
\text { Sarkodie y } \\
\text { Owusu (2017) }\end{array}$ \\
\hline Local & Pesos de los criterios & $\begin{array}{l}\text { Análisis de } \\
\text { incertidumbre y } \\
\text { sensibilidad espacial }\end{array}$ & $\begin{array}{l}\text { Ligmann- } \\
\text { Zielinska y } \\
\text { Jankowski (2014) }\end{array}$ \\
\hline Regional & $\begin{array}{l}\text { Áreas agrícolas, erosión del suelo, } \\
\text { pérdida de nutrientes y } \\
\text { contaminación del agua }\end{array}$ & $\begin{array}{l}\text { Modelo de la Perdida } \\
\text { de suelo y erosión }\end{array}$ & $\begin{array}{l}\text { Nowak y } \\
\text { Schneider (2017) }\end{array}$ \\
\hline Regional & $\begin{array}{l}\text { Sensibilidad ecológica, cobertura } \\
\text { de la vegetación, calidad del suelo, } \\
\text { ambiente atmosférico }\end{array}$ & Índice ecológico & $\begin{array}{l}\text { Yu y Xudong } \\
(2016)\end{array}$ \\
\hline
\end{tabular}




\section{Indicadores ambientales de vegetación}

La biodiversidad a nivel mundial se está perdiendo a un ritmo sin precedentes a pesar de que en las últimas décadas se ha avanzado en su conservación a escala global. Siendo la vegetación un elemento importante en la biodiversidad, ya que juega un papel fundamental para la conservación, protección del suelo, secuestro del carbono, entre otros. Por lo que, a través de los indicadores ambientales se busca conocer la diversidad de la vegetación y sus cambios a través del tiempo (Zolekar y Bhagat, 2015; Turner et al., 2016; Wen et al., 2017; Zhang y Xu, 2017).

De las metodologías analizadas Romero-Calcerrada y Luque (2006) en una escala espacial de análisis local evaluaron la biodiversidad de los paisajes en un bosque a partir de un análisis multicriterio que predijo la idoneidad de un hábitat con especies indicadoras basados en características ambientales y de vegetación, mediante la combinación de sensores remotos y datos de campo. El modelo es un método multicriterio cuantitativo utilizado para combinar diferente información de especies. El método depende de las especies indicadoras y condiciones de la biodiversidad de las áreas protegidas, definido por un rango de probabilidad de ocurrencia; a través, de una evaluación objetiva de la aptitud de hábitat.

Bredemeier et al. (2015) en una escala de análisis espacial regional determinaron el valor de la conservación de la naturaleza de diferentes hábitats en función de la riqueza de especies a partir de unidades territoriales y describieron sus efectos cuantitativos sobre la biodiversidad y la conservación de la naturaleza con el uso de diferentes escalas espacio-temporales. Donde la escala espacial de los indicadores permite la aplicación de un modelo mediante un sistema de información geográfica.

El modelo evaluó el nivel de conservación de los hábitats sobre la base de los efectos en el paisaje de la flora con indicadores de presión y modifica el valor de la conservación de la densidad de los campos de cultivo y la variación de los cambios de presión de la agricultura.

En tanto, Dyer et al. (2017) En una escala de análisis espacial regional, evaluaron la biodiversidad de especies y hábitats raros y amenazados a partir de un indicador basado en patrones espaciotemporales, que muestra la relación entre el estado ecológico de las áreas determinadas por especies únicas y definieron que una especie amenazada no establece la presión de dicha especie en el estado ecológico de la biodiversidad, ya que requieren de la evaluación y priorización a través del análisis espacial y temporal. Estableciendo que, los indicadores de impacto ambiental son importantes herramientas para predecir el progreso del desarrollo y lo cambios de uso del suelo.

Mientras que, Li et al. (2017) En una escala de análisis espacial local, de la combinación de la cobertura terrestre y tendencias de cambio de índice de vegetación normalizado identificaron el desempeño ecológico, basado en la cobertura del suelo donde los resultados demuestran un incremento evidente en estos cambios e indican que la restauración ecológica está experimentando desafíos cada vez mayores debido a la creciente actividad humana y al entorno ecológico frágil.

En tanto que, Wen et al. (2017) analizaron los cambios de la vegetación a partir de factores antropogénicos de densidad de población, restauración ecológica artificial y urbanización en una escala espacial de análisis regional, con la finalidad de determinar el impacto de las actividades humanas en la vegetación mediante datos de diferentes épocas de los cambios en el ecosistema. 
Analizando los patrones espacio-temporales, determinaron que los factores antropogénicos tienen impactos en la vegetación, así como en los factores climáticos. De la información de los indicadores espacio-temporal de la vegetación es de suma importancia para el estudio del ambiente, específicamente de la biodiversidad (Cuadro 3).

Cuadro 3. Indicadores ambientales de la vegetación.

\begin{tabular}{|c|c|c|c|}
\hline $\begin{array}{l}\text { Escala de } \\
\text { análisis* }\end{array}$ & Datos espaciales & Método empleado & Autor \\
\hline Regional & $\begin{array}{l}\text { Tipo de cultivo y } \\
\text { riqueza de especies de } \\
\text { flora }\end{array}$ & Modelos de unidades territoriales & $\begin{array}{l}\text { Bredemeier et al. } \\
(2015)\end{array}$ \\
\hline Regional & $\begin{array}{l}\text { Especies amenazadas, } \\
\text { patrones espaciales y } \\
\text { temporales }\end{array}$ & Modelo de biodiversidad & Dyer et al. (2017) \\
\hline Local & $\begin{array}{l}\text { Coberturas de } \\
\text { vegetación }\end{array}$ & $\begin{array}{l}\text { Análisis de cobertura terrestre y } \\
\text { tendencias de cambio del índice } \\
\text { de vegetación normalizado }\end{array}$ & Li et al. (2017) \\
\hline Local & Pesos & Análisis multicriterio & $\begin{array}{l}\text { Romero- } \\
\text { Calcerrada y } \\
\text { Luque }(2006)\end{array}$ \\
\hline Regional & $\begin{array}{l}\text { Cambios interanuales } \\
\text { de la vegetación }\end{array}$ & $\begin{array}{l}\text { Índice de vegetación (pp, } \mathrm{T}^{\mathrm{o}} \text {, } \\
\text { radiación) }\end{array}$ & Wen et al. (2017) \\
\hline
\end{tabular}

* Global, regional, local y paisaje.

En general, los cambios espaciales y temporales de los indicadores ambientales del agua, suelo y vegetación analizados presentan características similares en la necesidad de definir el área de estudio, modelo y temporalidad a analizar, aunado la importancia de contar con calidad y precisión de los datos.

\section{Análisis espacio-temporal de indicadores ambientales}

En las últimas décadas se han realizado enormes progresos en el análisis, caracterización y compresión de los procesos, funciones y estructuras del medio ambiente mediante indicadores ambientales. Sin embargo, muchos de los enfoques se basan en áreas de estudio de casos específicos y la transferencia de enfoques se ve obstaculizada debido a formatos de datos incompatibles y disponibles. Además, la modelización ambiental proporcionada por indicadores ambientales cambia continuamente, principalmente por los avances tecnológicos y metodológicos (Klug y Kmoch, 2015).

Por lo tanto, el desarrollo continuo de indicadores muestra la necesidad de nuevas y mejores formas de evaluar la situación actual de los recursos naturales de agua, suelo y vegetación, así como sintetizar información precisa y detallada (Vollmer et al., 2016) la cual cambia según las características espacio-temporales de la degradación ambiental y de las actividades humanas (Bao y Zou, 2018). 
A pesar de que existe una gran diversidad de artículos sobre indicadores ambientales espaciotemporales, no se ha logrado enfatizar en alguna metodología definitiva para el estudio de los recursos naturales. Por tanto, es necesario conocer diferentes metodologías para adaptar el problema ambiental que se enfrenta, así como conocer la importancia de las tecnologías y de la información disponible para el estudio del ambiente, ya que la comprensión adecuada de las características espacio-temporales de este atributo ambiental, es importante en la toma de decisiones para hacer frente a problemas ambientales mediante una buena gestión (Alphan, 2017).

Principalmente porque los indicadores son eficientes para evaluar los impactos de los recursos naturales ocasionado por las actividades de los seres humanos (Bredemeier et al., 2015; Alphan, 2017; Bao y Zou, 2018).

El tipo de análisis a utilizar como indicador ambiental deberá cuantificar diferentes variaciones, orientado a la gestión de la conservación y aprovechamiento de la naturaleza en la integración con la diversidad de hábitats y los recursos naturales (Ligmann-Zielinska y Jankowski, 2014; ArnaizSchmitz et al., 2017; Bao y Zou, 2018).

Un modelo ambiental de indicadores ambientales se aplica usualmente a unidades territoriales (espacial) en determinado escenario (temporal) con posibles efectos (Walz, 2015; Zolekar y Bhagat, 2015). Donde, el análisis espacio-temporales demuestran una gran variación de los recursos naturales (Schindler et al., 2015; Dye et al., 2017) tanto, dentro del área de estudio como de las zonas ambientales locales y regionales.

De las metodologías analizadas en este documento, se observó la necesidad de seguir trabajando en la generación de conocimientos apegados a la realidad para definir modelos predictivos confiables y comparables con la realidad (Romero-Calcerrada y Luque, 2006; Li et al., 2017).

Mediante, una evaluación objetiva de indicadores ambientales espacio-temporales para cada recurso natural que proporcione información sólida a través de la simulación de procesos y criterios para la toma de decisiones (Romero-Calcerrada y Luque, 2006; Ligmann-Zielinska and Jankowski, 2014) ya que, las formas de medir y equilibrar las necesidades y usos de los recursos naturales son esenciales para comprender y gestionarlos adecuadamente.

Con respecto a los indicadores ambientales examinados la mayoría se centra en los recursos naturales como escasos o muy demandados y con una gran dependencia de parte del ser humano. Por lo que, la credibilidad o el rigor científico y técnico de un indicador es el enfoque principal de la revisión, ya que los aspectos teóricos-metodológicos de la información científica, tecnológica y los valores sociales desempeñan un papel importante en la aplicación de cada indicador ambiental.

\section{Conclusiones}

Los indicadores ambientales monitorean, analizan e informan sobre las condiciones y los cambios ambientales mediante la integración interdisciplinaria a nivel local y regional de acuerdo con la información ambiental disponible de cada caso de estudio, para permite evaluar y facilitar la comprensión de la complejidad de la naturaleza; a través, de la cuantificación de los beneficios o impactos ambientales. 
Cada modelo ambiental de indicadores ambientales debe cumplir con normas nacionales e internacionales para garantizar la interoperabilidad, comparabilidad, transferibilidad e integración de la información, ya que existe una gran cantidad de indicadores ambientales en uso, pero una variación considerable en cuanto a lo que se mide y cómo se aplica cada indicador, por lo que dificulta que los usuarios finales identifiquen los métodos de evaluación adecuados.

Actualmente, con apoyo de las tecnologías, se tienen diferentes métodos para generar y analizar indicadores ambientales espacio-temporales con información puntual pero aún falta mucho para generar información que muestre la situación en tiempo real de cada problemática ambiental.

Los indicadores ambientales del agua, suelo y vegetación están definidos por los cambios espaciotemporales del área de estudio, la calidad y precisión de los datos a utilizar.

\section{Literatura citada}

Alphan, H. 2017. Analysis of landscape changes as an indicator for environmental monitoring. Environmental Monitoring and Assessment. 189(1):1-24. doi:10.1007/s10661-016-5748-7.

Arnaiz-Schmitz, C.; Schmitz, M. F.; Herrero-Jauregui, C.; Gutierrez-Angonese, J.; Pineda, F. D. and Montes, C. 2017. Identifying socio-ecological networks in rural-urban gradients: Diagnosis of a changing cultural landscape. Sci Total Environ. 612(1):625-635.

Asumadu-Sarkodie, S. and Owusu, P. A. 2017. The impact of energy, agriculture, macroeconomic and human-induced indicators on environmental pollution: evidence from Ghana. Environmental Science and Pollution Research. 24(7):6622-6633.

Azadeh, A.; Shafiee, F.; Yazdanparast, R.; Heydari, J. and Fathabad, A. M. 2017. Evolutionary multi-objective optimization of environmental indicators of integrated crude oil supply chain under uncertainty. J. Cleaner Produc. 152(1)295-311.

Bao, C. and Zou, J. 2018. Analysis of spatiotemporal changes of the human-water relationship using water resources constraint intensity index in Northwest China. Ecol. Indic. 84(1):119-129.

Bredemeier, B.; von Haaren, C.; Rüter, S.; Reich, M. and Meise, T. 2015. Evalligmanuating the nature conservation value of field habitats: A model approach for targeting agrienvironmental measures and projecting their effects. Ecol. Modelling. 295(1):113-122.

Dyer, R. J.; Gillings, S.; Pywell, R. F.; Fox, R.; Roy, D. B. and Oliver, T. H. 2017. Developing a biodiversity-based indicator for large-scale environmental assessment: a case study of proposed shale gas extraction sites in Britain. J. Appl. Ecol. 54(3):872-882.

Echavarren, J. M. 2017. From objective environmental problems to subjective environmental concern: a multilevel analysis using 30 indicators of environmental quality. Soc. Natural Res. 30(2):145-159.

Gao, X.; Zhao Q.; Zhao X.; Wu, P.; Pan, W.; Gao, X. and Sun, M. 2017. Temporal and spatial evolution of the standardized precipitation evapotranspiration index (SPEI) in the Loess Plateau under climate change from 2001 to 2050. Science of the Total Environment. 592(1):60-67. doi:10.1016/j.scitotenv.2017.03.226.

Klug, H. and Kmoch, A. 2015. Operationalizing environmental indicators for real time multipurpose decision making and action support. Ecological Modelling. 295(1):66-74. doi:10.1016/j.ecolmodel.2014.04.009. 
Li, Y.; Cao, Z.; Long, H.; Liu, Y. and Li, W. 2017. Dynamic analysis of ecological environment combined with land cover and NDVI changes and implications for sustainable urban-rural development: the case of Mu Us Sandy Land, China. J. Clean Produc. 142(1):697-715. doi:10.1016/j.jclepro.2016.09.011.

Ligmann-Zielinska, A. and Jankowski, P. 2014. Spatially-explicit integrated uncertainty and sensitivity analysis of criteria weights in multicriteria land suitability evaluation. Environ. Modell. Software. 57(1):235-247. doi:10.1016/j.envsoft.2014.03.007.

Luo, J.; Huai, W. X. and Gao, M. 2017. Indicators for environmental dispersion in a two-layer wetland flow with effect of wind. Ecol. Indic. 78(1):421-436. doi:10.1016/j.ecolind. 2017.03.027.

Neher, D. A.; Williams, K. M. and Lovell, S. T. 2017. Environmental indicators reflective of road design in a forested landscape. Ecosphere. 8(3):1-17. doi:e0173410.1002/ecs2.1734.

Nowak, A. and Schneider, C. 2017. Environmental characteristics, agricultural land use, and vulnerability to degradation in Malopolska Province (Poland). Sci Total Environ. 590591(1) :620-632. doi:10.1016/j.scitotenv.2017.03.006.

Park, Y. S.; Baehr, C.; Larocque, G. R.; Sánchez-Pérez, J. M. and Sauvage, S. 2015. Ecological modelling for ecosystem sustainability. Ecol. Modelling. 306(1): 1-5. doi:10.1016/ j.ecolmodel.2015.04.008.

Pratama, Y. W.; Purwanto, W. W.; Tezuka, T.; McLellan, B. C.; Hartono, D.; Hidayatno, A. and Daud, Y. 2017. Multi-objective optimization of a multiregional electricity system in an archipelagic state: The role of renewable energy in energy system sustainability. Renewable Sustainable Energy Reviews. 77(1):423-439. doi:10.1016/j.rser.2017.04.021.

Rodríguez M. L.; López B. J. y Vela C. G. 2013. Indicadores ambientales biofisicos a escala detallada para la planificación territorial en Milpa Alta, Centro México. Investigaciones Geográfica-Universida Nacional Autónoma de México (UNAM). Boletín del Instituto de Geografía, núm. 14. http://www.investigacionesgeograficas.unam.mx/index.php/rig/ article/view/36394/52842.

Romero-Calcerrada, R. and Luque, S. 2006. Habitat quality assessment using Weights-of-Evidence based GIS modelling: the case of Picoides tridactylus as species indicator of the biodiversity value of the finnish forest. Ecol. Modelling. 196(1-2):62-76. doi:10.1016/j.ecolmodel.2006.02.017.

Schindler, S.; von Wehrden, H.; Poirazidis, K.; Hochachka, W. M.; Wrbka, T. and Kati, V. 2015. Performance of methods to select landscape metrics for modelling species richness. Ecol. Modelling. 295(1):107-112. doi:10.1016/j.ecolmodel.2014.05.012.

Shrestha, S.; Farrelly, J.; Eggleton, M. and Chen, Y. 2017. Effects of conservation wetlands on stream habitat, water quality and fish communities in agricultural watersheds of the lower Mississippi River Basin. Ecol. Eng. 107(1):99-109. doi:10.1016/j.ecoleng.2017.06.054.

Sinha, S.; Chakraborty, S. and Goswami, S. 2017. Ecological footprint: an indicator of environmental sustainability of a surface coal mine. Environ. Develop. Sustainab. 19(3):807-824. doi:10.1007/s10668-016-9766.

Turner, K. G.; Anderson, S.; Gonzales-Chang, M.; Costanza, R.; Courville, S.; Dalgaard, T.; Dominati, E.; Kubiszewski, I.; Ogilvy, S.; Porfirio, L.; Ratna, N.; Sandhu, H.; Sutton, P. C.; Svenning, J. C.; Turner G. M.; Varennes Y. D.; Voinov A. and Wratten, S. 2016. A review of methods, data, and models to assess changes in the value of ecosystem services from land degradation and restoration. Ecol. Modelling. 319(1):190-207. doi:10.1016/j.ecolmodel.2015.07.017. 
Vollmer, D.; Regan, H. M. and Andelman, S. J. 2016. Assessing the sustainability of freshwater systems: A critical review of composite indicators. Ambio. 45(7):765-780. doi:10.1007/s13280-016-0792-7.

Walz, U. 2015. Indicators to monitor the structural diversity of landscapes. Ecol. Modelling. 295(1):88-106. doi:10.1016/j.ecolmodel.2014.07.011.

Wen, Z.; Wu, S.; Chen, J. and Lu, M. 2017. NDVI indicated long-term interannual changes in vegetation activities and their responses to climatic and anthropogenic factors in the Three Gorges Reservoir Region, China. Sci. Total Environ. 574(1):947-959. doi:10.1016/j.scitotenv.2016.09.049.

WWAP. 2017. Informe sobre el desarrollo de los recursos hídricos de las naciones unidades: cifras y datos. Las aguas residuales. El recurso desaprovechado. UNESCO. http://unesdoc.unesco.org/images/0024/002476/247647s.pdf.

$\mathrm{Xu}$, E. and Zhang, H. 2013. Spatially-explicit sensitivity analysis for land suitability evaluation. Appl. Geo. 45(1):1-9. doi:10.1016/j.apgeog.2013.08.005.

$\mathrm{Yu}, \mathrm{Z}$. and Xudong, C. 2016. A study on the choices of construction land suitability evaluation of ecological index. Procedia Computer Sci. 91(1):180-183. doi:10.1016/j.procs.2016.07.054.

Zhang, H. and Xu, E. 2017. An evaluation of the ecological and environmental security on China's terrestrial ecosystems. Sci Rep. 7(811):1-12. doi:10.1038/s41598-017-00899-X.

Zolekar, R. B. and Bhagat, V. S. 2015. Multi-criteria land suitability analysis for agriculture in hilly zone: remote sensing and GIS approach. Computers and Electronics in Agriculture. 118(1):300-321. doi:10.1016/j.compag.2015.09.016. 